\title{
Ring Formation in the Disk of Barred Galaxies
}

\author{
B. Guivarch and E. Athanassoula \\ Observatoire de Marseille, 2 place Leverrier, 13248 Marseille Cedex 4, \\ France
}

\begin{abstract}
We study the effects of different collision laws and of recycling on the results of simulations using sticky particles to describe the gas clouds in a disc galaxy.
\end{abstract}

Schwarz (1981) introduced a simple method for performing hydrodynamical simulations by representing the gas clouds in a disc galaxy by colliding particles, which at every collision lose some of their kinetic energy. Its success in explaining the formation of rings in barred galaxies, together with its simplicity, made this model very popular and it has been since widely used under the name of "sticky particles". Nevertheless we found it quite inadequate for modeling the strong offset shocks found on the leading edges of some barred galaxies, which other hydrodynamical methods can describe in a very satisfactory manner (cf. Athanassoula 1992), and for this reason we have tried to understand the effect of different collision laws on the results. Here we concentrate on the formation of outer rings.

Figure 1 shows the response of an ensemble of gas clouds to the potential used by Schwarz (1981), where collision laws identical to those of Schwarz are used. The only difference is that we use 10000 instead of 2000 clouds so that more details are seen in the plots.

Figure 2 shows the response of 10000 particles to the same potential as before, with two differences however. The first concerns the way the colliding pairs are chosen. Schwarz drew them at random from particles within a cell of a cartesian grid. This has the disadvantage that a given particle may not collide with its nearest neighbor if the latter is in an adjacent cell, but may collide with a particle much further away provided it is in the same cell. We, on the other hand, consider collision with the nearest neighbor, and, to speed up calculations, search for them in the cell containing the particles under consideration and the eight adjacent cells. The second difference is that we introduced recycling, taking gas from high density regions (to mimic star formation) and redistribute it at random on the disc (mimicking mass loss from stars). Such a recycling scheme has also been used in the hydrodynamic codes (e.g. Athanassoula 1992). We note that there are non-negligible differences between the final results of the two simulations: with the new method the ring is much thinner and, instead of the intricate pattern around the Lagrangian points, one sees the last traces of the spiral, together with a rectangular-like density enhancement around the bar region.

Figure 3 shows the end result of four simulations with the same form of model potential but a relatively stronger bar. The upper left panel was obtained using Schwarz's method, the upper right one with Schwarz's collision law but 


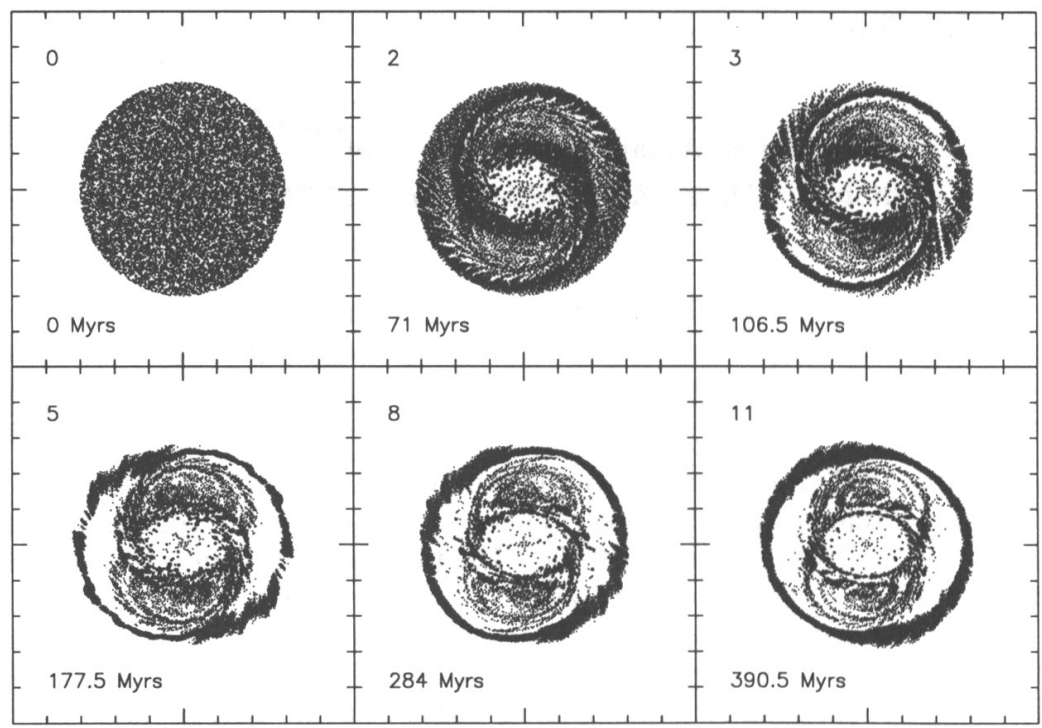

Figure 1. Evolution of the response of gas clouds to a barred potential when Schwarz's method is used. Time is given in bar rotation periods in the upper left corner of each frame.

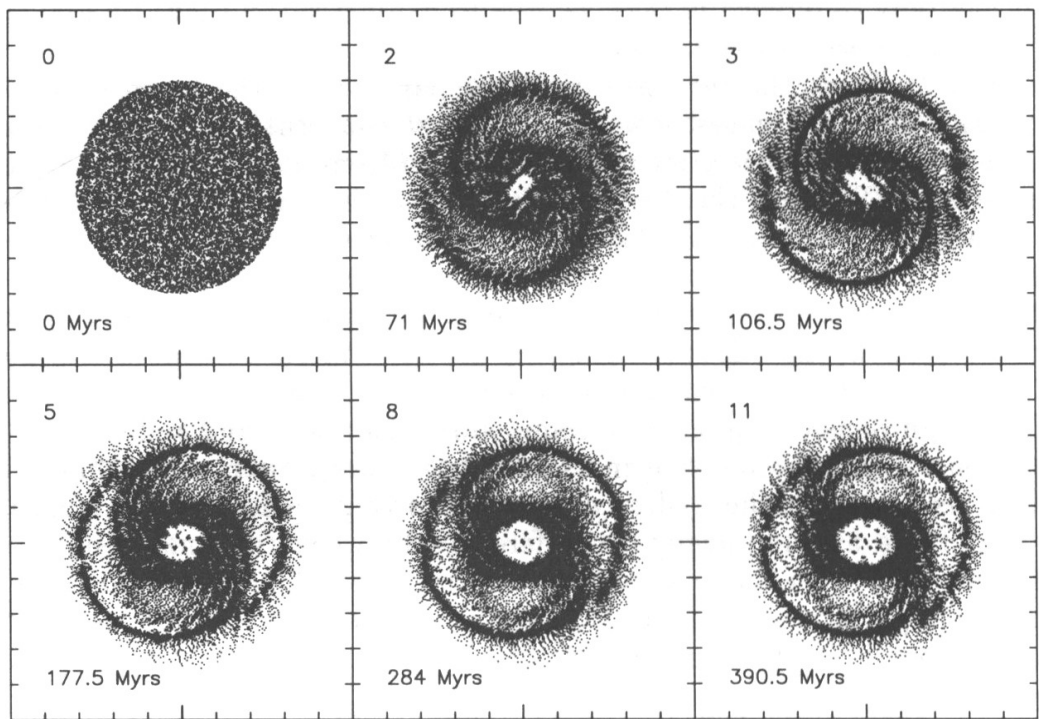

Figure 2. As in Figure 1, but with the collision laws and recycling described in the text. 


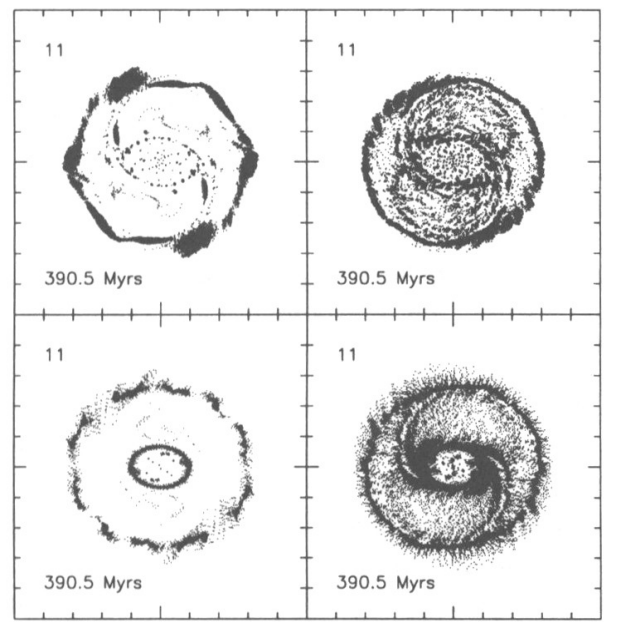

Figure 3. Result of the response to a stronger bar after 11 bar rotations (see text).

including recycling; the lower left panel corresponds to our collision law with no recycling, and the lower right one to our collision law with recycling. As expected recycling allows the structures to last for longer times. The lower left panel contains the same number of points as the others, the impression that there are less comes only from the fact that they are more concentrated in the lumps. The important thing to note is the difference between the two right hand panels. For our collision laws the ring is narrower and the spiral as well as the bar region are much better defined.

We have also tried the effects of different bar lengths, strengths and pattern speeds, as well as other differences in the collision laws. These more complete results will be presented elsewhere.

Acknowledgments. We would like to thank Albert Bosma for many interesting discussions and suggestions.

\section{References}

Athanassoula, E. 1992, MNRAS, 259, 345

Schwarz, M. P. 1981, ApJ, 247, 77 